\title{
Iron and carbon monoxide enhance coagulation and attenuate fibrinolysis by different mechanisms Vance G. Nielsen ${ }^{\mathrm{a}}$ and Etheresia Pretorius ${ }^{\mathrm{b}}$
}

\author{
The Department of Anesthesiology, The University of Arizona College of Medicine, Tucson, Arizona, USA and ${ }^{\text {}}$ Faculty of Health Sciences, Department of Physiology, \\ University of Pretoria, Arcadia, South Africa \\ Correspondence to Vance G. Nielsen, MD, Department of Anesthesiology, The University of Arizona College of Medicine, PO Box 245114, 1501 North Campbell \\ Avenue, Tucson, AZ 85724-5114, USA \\ Tel: +1 520626 7195; fax: +1 520626 6943; e-mail: vgnielsen333@gmail.com
}

\begin{abstract}
Two parallel lines of investigation elucidating novel mechanisms by which iron (scanning electron microscopy-based) and carbon monoxide (viscoelastic-based) enhance coagulation and diminish fibrinolysis have emerged over the past few years. However, a multimodal approach to ascertain the effects of iron and carbon monoxide remained to be performed. Such investigation could be important, as iron and carbon monoxide are two of the products of heme catabolism via heme oxygenase-1, an enzyme upregulated in a variety of disease states associated with thrombophilia. Human plasma was exposed to ferric chloride, carbon monoxide derived from carbon monoxide-releasing molecule-2, or their combination. Viscoelastic studies demonstrated ferric chloride and carbon monoxide mediated enhancement of velocity of growth, and final clot strength, with the combination of the two molecules noted to have all the prothrombotic kinetic effects of either separately. Parallel ultrastructural studies demonstrated separate types of fibrin polymer cross-linking and matting in plasma exposed to ferric chloride and carbon monoxide, with the combination sharing features of each molecule. In conclusion, we present the first evidence that iron and carbon monoxide interact with key coagulation and fibrinolytic processes, resulting in thrombi that begin to form more quickly, grow faster, become stronger, and are more resistant to lysis.
\end{abstract}

Keywords: carbon monoxide, coagulation, fibrinogen, fibrinolysis, iron

\section{Introduction}

Two parallel lines of investigation elucidating novel mechanisms that modulate both coagulation and fibrinolysis have emerged over the past few years. First, it was posited, and then determined that iron modified fibrinogen, which enhanced coagulation and attenuated fibrinolysis as documented by spectrophotometric and scanning electron micrographic (SEM) techniques [17]. Second, it was serendipitously discovered that carbon monoxide enhanced fibrinogen-dependent coagulation via an associated heme(s) group and attenuated fibrinolysis via upregulation of $\alpha_{2}$-antiplasim activity and downregulation of plasmin activity via enzyme-associated heme(s) [8-14]. Additional works validated the procoagulant efficacy of exogenous carbon monoxide via administration of carbon monoxide-releasing molecule2 (CORM-2) in rabbit models involving anticoagulation with antiplatelet medications or administration of tissuetype plasminogen activator [15-16]. While by themselves interesting, the hemostatic impact of increased systemic concentrations of iron and carbon monoxide likely occurs in vivo, as they are both products of the catalytic action of heme oxygenase-1 (HO-1) [17].

Upregulation of HO- 1 occurs in a variety of situations that involve red blood cell lysis and release of heme, such as in the case of biomaterial-blood interaction during mechanical circulatory support [18]. Catalytically released iron may also contribute to hydroxyl radical modification of fibrinogen [19] or bind directly to fibrinogen [20] as recently demonstrated. HO-1 activity is also increased systemically in inflammatory disorders associated with thrombophilia, such as diabetes mellitus [21] and rheumatoid arthritis [22]. Critically, SEM-based investigations demonstrated that the fibrin matrix formed from blood obtained from individuals with diabetes mellitus [23] or rheumatoid arthritis [24] was very similar to that observed when normal blood was exposed to ferric chloride [2-7]. In a complementary fashion, using a viscoelastic methodology validated to detect carbon monoxide-mediated hypercoagulability in the setting of tobacco smoking [25], it was determined that a patient with a clotted ventricular assist device and hemolysis had carbon monoxide-mediated hypercoagulability, and upregulation of HO-1 was documented by increased carboxyhemoglobin concentrations [26]. Similarly, in a series of works, upregulation of HO-1 and carbon monoxide-mediated hypercoagulability have been documented in individuals with thyroid [27], breast [28], brain [29], thoracic [30], colon [31], and pancreas [31] cancers. Considered as a whole, these various investigations partially characterize the SEM and viscoelastic consequences of increased iron and carbon monoxide concentrations during HO-1 upregulation, but not in an integrated 
and comprehensive manner. Unfortunately, critical gaps in mechanistic insight into the hemostatic impact of interaction of iron and carbon monoxide remained to be elucidated.

Thus, the purpose of this investigation was to combine both SEM and viscoelastic methodologies and determine the ultrastructural consequences and coagulation/fibrinolytic kinetic profiles of iron and carbon monoxide, separately and in combination.

\section{Methods}

\section{Viscoelastic-based analyses}

All viscoelastic-based experiments were performed at the University of Arizona. Frozen, citrate anticoagulated normal pooled plasma and plasma collected from an anonymous factor XIII (FXIII)-deficient donor were obtained from a commercial vendor (George King BioMedical, Overland Park, Kansas, USA) for use in subsequently described experimentation. As determined by the vendor, the normal pooled plasma had a prothrombin time of $13.7 \mathrm{~s}$ (normal 12.8-14.8 s), a partial thromboplastin time of $31.6 \mathrm{~s}$ (normal 26.3-35.9s), coagulation factor II, V, VII, VIII, IX, X, XI, and XII activity at least $96 \%$ of normal, and fibrinogen concentration at least $250 \mathrm{mg} / \mathrm{dl}$. The FXIII-deficient plasma had prothrombin time of $13.4 \mathrm{~s}$, an activated partial thromboplastin time of $33.6 \mathrm{~s}$, and a FXIII activity less than $5 \%$ of normal. With regard to chemicals utilized, ferric chloride $\left(\mathrm{FeCl}_{3}\right.$, 99.9\% pure), CORM-2 (tricarbonyldichlororuthenium (II) dimer), dimethyl sulfoxide, and calcium-free phosphatebuffered saline (PBS) were obtained from a commercial vendor (Sigma-Aldrich, Saint Louis, Missouri, USA).

Coagulation kinetic investigations were conducted as follows. Both normal pooled plasma and FXIII-deficient plasma were used in this series of experiments. Plasma was rapidly thawed at $37^{\circ} \mathrm{C}$ on the day of experimentation. The final volume for all subsequently described plasma sample mixtures was $359.6 \mu \mathrm{l}$. Sample composition consisted of $332 \mu \mathrm{l}$ of plasma; $3.6 \mu \mathrm{l}$ of PBS or $\mathrm{FeCl}_{3}\left(10 \mu \mathrm{mol} / \mathrm{l}\right.$ final concentration); $3.6 \mu \mathrm{l}$ of $\mathrm{dH}_{2} \mathrm{O}$ or CORM-2 (100 $\mu \mathrm{mol} / \mathrm{l}$ final concentration); and $20 \mu \mathrm{l}$ of $200 \mathrm{mmol} / 1 \mathrm{CaCl}_{2}$. These concentrations of $\mathrm{FeCl}_{3}[2,3]$ and CORM-2 $[8,12]$ were chosen as they have been demonstrated to maximally enhance coagulation in our plasma and whole blood systems. Plasma sample mixtures were placed in a disposable cup in a computercontrolled thrombelastograph hemostasis system (Model 5000; Haemoscope Corp., Niles, Illinois, USA), with addition of $\mathrm{CaCl}_{2}$ after a 3-min incubation period as the last step to initiate clotting. Data were collected at $37^{\circ} \mathrm{C}$ until maximum amplitude was obtained. Elastic modulus-based parameters previously described were determined $[8,12]$. Specifically, the parameters measured were as follows: time to maximum rate of thrombus generation (TMRTG) - this is the time interval (min) observed prior to maximum speed of clot growth; maximum rate of thrombus generation (MRTG) - this is the maximum velocity of clot growth observed (dynes/ $\mathrm{cm}^{2} / \mathrm{s}$ ); total thrombus generation (TTG) - this is the total area under the velocity curve during clot growth (dynes $/ \mathrm{cm}^{2}$ ), representing the amount of clot strength generated during clot growth; and clot growth time (CGT) - defined by the time (min) clot formation commences ( $2 \mathrm{~mm}$ amplitude) until growth ceases (maximum amplitude obtained).

Fibrinolytic kinetic investigations were conducted as follows. Only normal pooled plasma was utilized in these experiments. Plasma was rapidly thawed at $37^{\circ} \mathrm{C}$. The final volume for all subsequently described plasma sample mixtures was $359.6 \mu \mathrm{l}$. Sample composition consisted of $322 \mu \mathrm{l}$ of plasma; $10 \mu \mathrm{l}$ of tissue type plasminogen activator (tPA, $580 \mathrm{IU} / \mu \mathrm{g}$; Genentech Inc., San Francisco, California, USA; $100 \mathrm{IU} / \mathrm{ml}$ final concentration); $3.6 \mu \mathrm{l}$ of $\mathrm{PBS}$ or $\mathrm{FeCl}_{3}(10 \mu \mathrm{mol} / \mathrm{l}$ final concentration); $3.6 \mu \mathrm{l}$ of $\mathrm{dH}_{2} \mathrm{O}$ or CORM-2 $(100 \mu \mathrm{mol} / \mathrm{l}$ final concentration); and $20 \mu \mathrm{l}$ of $200 \mathrm{mmol} / \mathrm{l} \mathrm{CaCl}_{2}$. As with the previous series of experiments, sample mixtures were placed in a disposable cup in a computer-controlled thrombelastograph hemostasis system, with addition of $\mathrm{CaCl}_{2}$ after a 3 -min incubation period as the last step to initiate clotting. Data were collected at $37^{\circ} \mathrm{C}$ until clot lysis time (CLT) was observed. In addition to determining the aforementioned growth parameters, additional kinetic determinants were measured: time to maximum rate of lysis (TMRL) - defined as the time when maximum amplitude is observed until the time (min) of maximum velocity of clot lysis is observed; maximum rate of lysis (MRL) - the greatest velocity of clot lysis (dynes $/ \mathrm{cm}^{2}$ per s); CLT - defined as the time ( $\mathrm{min}$ ) from when growth ceases until lysis is complete (amplitude returns to $2 \mathrm{~mm}$ ); clot lifespan (CLS) - defined as the sum of CGT and CLT. These elastic modulus-based parameters of thrombus fibrinolytic kinetics have been previously described in detail [12].

\section{Scanning electron micrographic-based analyses}

All SEM-based analyses were conducted at the University of Pretoria. The Institutional Review Board of the University of Pretoria granted approval for healthy individuals for SEM-based investigations [ethics number $151 / 2006$ (E.P.) that is extended until end of 2014]. Whole blood was collected from three healthy, female patients after obtaining written informed consent. They were nonsmokers, aged 25, 26, and 44 years of age. Their serum ferritin (range 13-28 ng/ml) and percentage of iron saturation of ferritin (range 19-33\%) were within normal range. As previously presented [23,24,32], $40 \mathrm{ml}$ of whole blood was collected and anticoagulated with sodium citrate (nine parts blood to one part $0.105 \mathrm{~mol} / \mathrm{l}$ sodium citrate), and platelet-rich plasma (PRP) was obtained from each collected sample by centrifuging the whole blood at $1250 \mathrm{~g}$ for $2 \mathrm{~min}$. 
Using a methodology previously noted [23,24,32], PRP smears with the addition of different compounds (four conditions) were made on glass cover slips and incubated for $8 \mathrm{~min}$ at $37^{\circ} \mathrm{C}$. The four conditions were as follows: $10 \mu \mathrm{l}$ PRP without additions and $5 \mu \mathrm{l}$ thrombin $(10 \mathrm{U} / \mathrm{ml})$, mixed and incubated for $3 \mathrm{~min} ; 10 \mu \mathrm{PRP}$ with $5 \mu \mathrm{FeCl}_{3}$ ( $250 \mu \mathrm{mol} / \mathrm{l}$ final concentration) added and then addition of $5 \mu \mathrm{l}$ thrombin $(10 \mathrm{U} / \mathrm{ml})$ mixed and incubated for $3 \mathrm{~min} ; 10 \mu \mathrm{l}$ PRP after $1 \%$ addition ( $\mathrm{v} / \mathrm{v})$ of CORM-2 $(10 \mu \mathrm{mol} / \mathrm{l}$ final concentration in PRP, suspended in PBS with final concentration of $0.1 \%$ dimethyl sulfoxide; PRP was then incubated for $5 \mathrm{~min}$ ) with addition of $5 \mu \mathrm{l}$ of thrombin $(10 \mathrm{U} / \mathrm{ml})$, mixed and incubated for $3 \mathrm{~min}$; and $10 \mu \mathrm{l}$ PRP after simultaneous $1 \%$ addition $(\mathrm{v} / \mathrm{v})$ of CORM-2 $(10 \mu \mathrm{mol} / \mathrm{l}$ final concentration in PRP, incubated for $5 \mathrm{~min}$ ) with $1 \%$ addition $(\mathrm{v} / \mathrm{v})$ of $\mathrm{FeCl}_{3}$ ( $333 \mu \mathrm{mol} / \mathrm{l}$ final concentration), and then addition of $5 \mu \mathrm{l}$ thrombin $(10 \mathrm{U} / \mathrm{ml})$, mixed and incubated for 3 min. The concentration of $\mathrm{FeCl}_{3}$ for condition 2 was used for simplicity, as there is little difference in thrombus ultrastructure with concentrations between $6 \mu \mathrm{mol} / \mathrm{l}$ and nearly $4 \mathrm{mmol} / 1[3,6]$. The concentration of CORM-2 used was smaller than that used in the viscoelastic studies to maximize detection of more subtle changes in thrombus ultrastructure, without and with a lower concentration of $\mathrm{FeCl}_{3}$. The cover slips were then placed in $0.075 \mathrm{~mol} / \mathrm{l} \mathrm{PBS}$ on a shaker and washed for $20 \mathrm{~min}$. The samples were then fixated for $30 \mathrm{~min}$ followed by three washing steps in $0.075 \mathrm{~mol} / \mathrm{l} \mathrm{PBS}$ for $3 \mathrm{~min}$ to remove any residual fixative. The smears were then postfixated for 15 min with $1 \%$ osmium tetroxide, followed by a washing process, for $3 \mathrm{~min}$ in $0.075 \mathrm{~mol} / \mathrm{l} \mathrm{PBS}$. The samples were finally dehydrated serially in 30,50,70, and $90 \%$ and then three times in $100 \%$ ethanol followed by drying using hexamethyldisilazane; mounting; and coating with carbon. Once the samples had been coated, they were examined using a scanning electron microscope (Zeiss ULTRA plus FEG SEM; Carl Zeiss Microscopy GmbH, Jena, Germany). All three normal individuals had images obtained for each condition, and representative microphotographs were recorded for presentation.

\section{Statistical analyses and graphics}

Viscoelastic data are presented as mean $\pm S D$, with analyses conducted with a commercially available statistical program (SigmaStat 3.1; Systat Software Inc., San Jose, California, USA). All viscoelastic experiments had $n=8$ replicates per condition, as this number of replicates provides statistical power more than 0.8 in these systems with the variability typically observed [8,10-14]. Graphics were generated with a commercially available program (OrigenPro 7.5; OrigenLab Corporation, Northampton, Massachusetts, USA). The analyses of the coagulation and fibrinolytic kinetic effects of addition of $\mathrm{FeCl}_{3}$ and CORM-2 to normal and FXIIIdeficient plasma were conducted with one-way analysis of variance with the Holm-Sidak post-hoc test. A $P$ value $<0.05$ was considered significant. Micrographs of SEM data were generated with Adobe Photoshop CS6 (Adobe Systems Inc., San Jose, California, USA).

\section{Results \\ Viscoelastic data}

The results of the experiments involving coagulation kinetics are displayed in Table 1, with representative thrombus velocity growth curves of the four conditions presented in Fig. 1. In normal plasma, exposure to $\mathrm{FeCl}_{3}$ resulted in a significant decrease in TMRTG compared with unexposed plasma, whereas CORM-2 exposure resulted in a significant increase in TMRTG compared with unexposed and ferric chloride-exposed plasma. However, the combination of $\mathrm{FeCl}_{3}$ and CORM-2 resulted in TMRTG values not significantly different from $\mathrm{FeCl}_{3}$ exposure alone. With regard to the velocity of thrombus growth, $\mathrm{FeCl}_{3}$ or CORM-2 exposure resulted in MRTG values significantly greater than unexposed plasma. Of interest, the MRTG values of samples exposed to both $\mathrm{FeCl}_{3}$ and CORM-2 were significantly greater than all other conditions, with an apparent additive effect present. With regard to clot strength, $\mathrm{FeCl}_{3}$ exposure did not significantly affect TTG compared with unexposed normal plasma. However, CORM-2 addition significantly increased TTG compared with all other

Table 1 Effects of $\mathrm{FeCl}_{3}$ and carbon monoxide-releasing molecule-2 on coagulation kinetics in normal and FXIII-deficient plasma

\begin{tabular}{|c|c|c|c|c|}
\hline Parameter & Vehicle & $\mathrm{FeCl}_{3}$ & CORM-2 & $\mathrm{FeCl}_{3}$ and CORM-2 \\
\hline \multicolumn{5}{|c|}{ Normal plasma } \\
\hline TMRTG & $15.9 \pm 2.6$ & $6.4 \pm 0.2^{*}$ & $18.1 \pm 0.5^{*, \dagger}$ & $6.9 \pm 0.9^{*, \ddagger}$ \\
\hline MRTG & $3.1 \pm 0.4$ & $8.0 \pm 0.8^{*}$ & $4.4 \pm 0.8^{*, \dagger}$ & $12.1 \pm 1.7^{*, t, \ddagger}$ \\
\hline TTG & $198 \pm 16$ & $192 \pm 17$ & $336 \pm 24^{*, \dagger}$ & $305 \pm 19^{*, \dagger} \ddagger$ \\
\hline CGT & $10.1 \pm 1.4$ & $7.8 \pm 0.8^{*}$ & $13.1 \pm 1.2^{*, \dagger}$ & $9.3 \pm 1.1^{\dagger, \ddagger}$ \\
\hline \multicolumn{5}{|c|}{ FXIII-deficient plasma } \\
\hline TMRTG & $25.7 \pm 4.2$ & $11.0 \pm 2.6^{*}$ & $21.0 \pm 2.1^{*, \dagger}$ & $9.1 \pm 0.5^{*, \ddagger}$ \\
\hline MRTG & $0.5 \pm 0.2$ & $2.4 \pm 1.0^{*}$ & $3.4 \pm 1.2^{*}$ & $10.6 \pm 1.6^{*, t, \ddagger}$ \\
\hline TTG & $64 \pm 11$ & $85 \pm 5^{*}$ & $291 \pm 15^{*, \dagger}$ & $284 \pm 17^{*, \dagger}$ \\
\hline CGT & $11.4 \pm 1.8$ & $5.4 \pm 2.3^{*}$ & $14.7 \pm 4.0^{*, \dagger}$ & $8.4 \pm 0.6^{*, \dagger, \ddagger}$ \\
\hline
\end{tabular}

Data are presented as mean $\pm \mathrm{SD}$. Vehicle $=$ phosphate buffered saline (PBS) and dimethyl sulfoxide $(\mathrm{DMSO})$ only; $\mathrm{FeCl}_{3}=10 \mu \mathrm{mol} / \mathrm{I} \mathrm{FeCl}{ }_{3} ; \mathrm{carbon}_{\mathrm{monoxide}} \mathrm{releasing}$ molecule-2 (CORM-2) =100 $\mu \mathrm{mol} / \mathrm{l} \mathrm{CORM-2.} \mathrm{CGT,} \mathrm{clot} \mathrm{growth} \mathrm{time} \mathrm{(min);} \mathrm{MRTG,} \mathrm{maximum} \mathrm{rate} \mathrm{of} \mathrm{thrombus} \mathrm{generation,} \mathrm{the} \mathrm{maximum} \mathrm{velocity} \mathrm{of} \mathrm{clot} \mathrm{growth} \mathrm{observed}$ (dynes/cm ${ }^{2}$ per s); TMRTG, time to maximum rate of thrombus generation, the time interval (min) observed prior to maximum speed of clot growth; TTG, total thrombus generation, the total area under the velocity curve during clot growth (dynes/cm ${ }^{2}$ ). ${ }^{*} P<0.05$ vs. vehicle. ${ }^{\dagger} P<0.05$ vs. $\mathrm{FeCl} 3 .{ }^{\ddagger} P<0.05$ vs. $\mathrm{CORM}-2$. 
Fig. 1

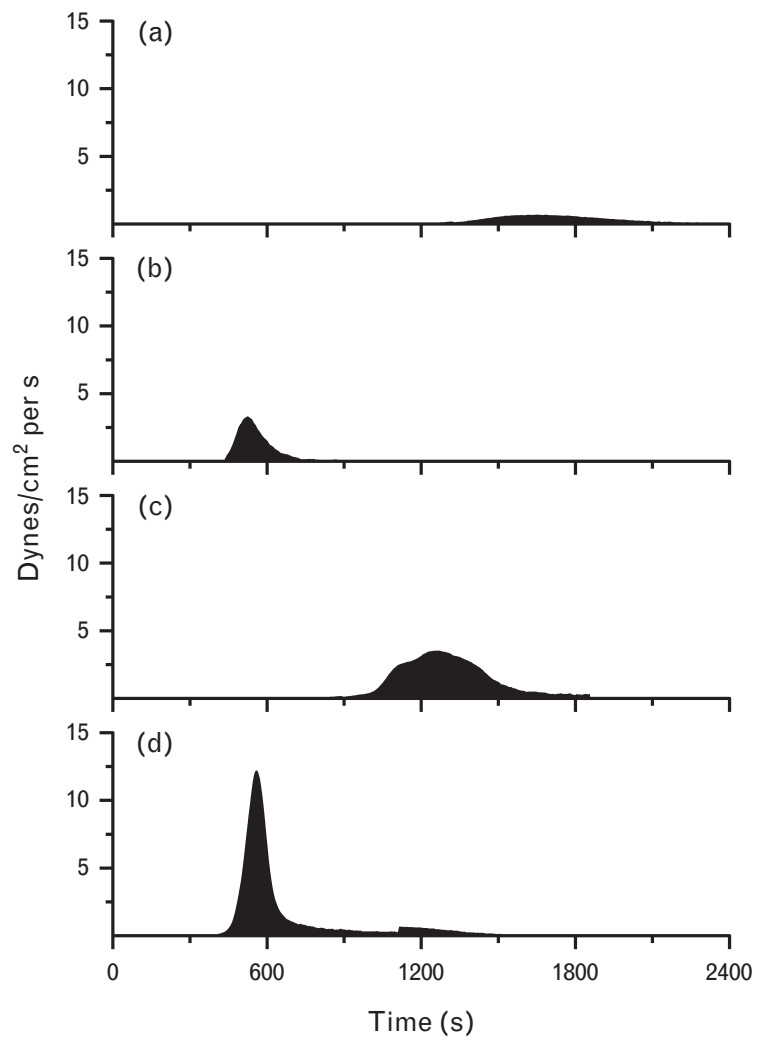

Effects of $\mathrm{FeCl}_{3}$ and carbon monoxide-releasing molecule-2 (CORM-2) on coagulation kinetics in FXIII-deficient plasma. Plasma was exposed to (a) phosphate buffered saline (PBS) and dimethyl sulfoxide (DMSO),

(b) DMSO and $10 \mu \mathrm{mol} / / \mathrm{FeCl}_{3}$, (c) PBS and $100 \mu \mathrm{mol} / \mathrm{l} \mathrm{CORM-2,} \mathrm{or}$ (d) $10 \mu \mathrm{mol} / / \mathrm{FeCl}_{3}$ and $100 \mu \mathrm{mol} / / \mathrm{CORM}-2$. Exposure to $\mathrm{FeCl}_{3}$ decreased the time to maximum speed of clot formation, increased the speed of clot formation, and increased clot strength. Addition of CORM-2 decreased the time to maximum speed of clot formation, increased the speed of clot formation, and markedly increased clot strength. The combination of $\mathrm{FeCl}_{3}$ and CORM-2 resulted in decreased time to maximum speed of clot formation not different from ferric chloride alone; the maximum speed of clot formation was double the summation of $\mathrm{FeCl}_{3}$ and CORM-2; and the final clot strength was not different than that of CORM-2 alone.

conditions, with a small but significant decrease in TTG noted when both $\mathrm{FeCl}_{3}$ and CORM-2 were added. Finally, with regard to the time taken for clot growth, $\mathrm{FeCl}_{3}$ exposure resulting in significantly decreased CGT values and CORM-2 exposure resulted in significantly prolonged CGT values compared with unexposed plasma. Interestingly, the combination of $\mathrm{FeCl}_{3}$ and CORM-2 resulted in CGT not different from unexposed plasma.

In FXIII-deficient plasma, exposure to $\mathrm{FeCl}_{3}$ or $\mathrm{CORM}$ 2 resulted in a significant decrease in TMRTG compared with unexposed plasma, whereas a combined exposure of $\mathrm{FeCl}_{3}$ and CORM-2 exposure resulted in TMRTG values significantly smaller than unexposed or CORM2 exposed plasma. As for the velocity of thrombus growth,
$\mathrm{FeCl}_{3}$ or CORM-2 exposure resulted in MRTG values significantly greater than unexposed plasma. Critically, the MRTG values of samples exposed to both $\mathrm{FeCl}_{3}$ and CORM-2 were significantly greater than all other conditions, with the resultant MRTG mean value being double of the sum of MRTG values of $\mathrm{FeCl}_{3}$ and CORM-2-exposed samples. With regard to clot strength, $\mathrm{FeCl}_{3}$ or CORM-2 exposure significantly increased TTG compared with unexposed normal plasma. However, although TTG values of samples exposed to both $\mathrm{FeCl}_{3}$ and CORM-2 addition had significantly increased TTG values compared with unexposed or $\mathrm{FeCl}_{3}$-exposed plasma, there was no significant difference from plasma exposed to CORM-2 alone. Finally, with regard to the time taken for clot growth, $\mathrm{FeCl}_{3}$ exposure resulting in significantly decreased CGT values and CORM-2 exposure resulted in significantly prolonged CGT values compared with unexposed plasma. Of interest, the combination of $\mathrm{FeCl}_{3}$ and CORM-2 resulted in CGT values significantly smaller than unexposed or CORM-2exposed plasma.

The results of the experiments involving fibrinolytic kinetics are displayed in Table 2, with representative thrombus velocity growth curves of the four conditions presented in Fig. 2. With regard to TMRTG, exposure to $\mathrm{FeCl}_{3}$ or CORM-2, or the two chemicals in combination, resulted in values significantly less than those observed in unexposed plasma. Similarly, exposure to $\mathrm{FeCl}_{3}$ or CORM-2 resulted in MRTG values significantly larger than unexposed plasma, with the combination of $\mathrm{FeCl}_{3}$ and CORM-2 resulting in MRTG values significantly greater than the other three conditions that were equivalent to the summation of MRTG values observed in $\mathrm{FeCl}_{3}$ or CORM-2 exposures separately. With regard to clot strength, exposure of plasma to $\mathrm{FeCl}_{3}$ or CORM-2 resulted in significantly greater TTG values than that observed with unexposed plasma; however, the

Table 2 Effects of $\mathrm{FeCl}_{3}$ and carbon monoxide-releasing molecule2 on fibrinolytic kinetics in normal plasma

\begin{tabular}{lcccc} 
Parameter & Vehicle & \multicolumn{1}{c}{$\mathrm{FeCl}_{3}$} & $\mathrm{CORM}-2$ & $\mathrm{FeCl}_{3}$ and CORM-2 \\
\hline TMRTG & $15.6 \pm 1.6$ & $6.3 \pm 0.2^{*}$ & $13.6 \pm 1.9^{*, \dagger}$ & $6.3 \pm 0.2^{*, \ddagger}$ \\
MRTG & $2.3 \pm 0.4$ & $7.6 \pm 0.8^{*}$ & $5.2 \pm 0.8^{*, \dagger}$ & $11.8 \pm 1.2^{*, \dagger, \ddagger}$ \\
TTG & $119 \pm 12$ & $163 \pm 12^{*}$ & $309 \pm 18^{*, \dagger}$ & $297 \pm 20^{*, \dagger}$ \\
TMRL & $7.8 \pm 3.6$ & $19.7 \pm 2.6^{*}$ & $42.2 \pm 2.5^{*, \dagger}$ & $37.8 \pm 2.0^{*, \dagger, \ddagger}$ \\
MRL & $0.8 \pm 0.1$ & $1.2 \pm 0.1^{*}$ & $1.5 \pm 0.1^{*, \dagger}$ & $1.6 \pm 0.1^{*, \dagger, \ddagger}$ \\
CGT & $5.9 \pm 1.1$ & $5.4 \pm 1.1$ & $11.0 \pm 0.4^{*, \dagger}$ & $8.7 \pm 1.1^{*, \dagger, \ddagger}$ \\
CLT & $17.8 \pm 2.6$ & $28.2 \pm 4.0^{*}$ & $61.2 \pm 3.3^{*, \dagger}$ & $59.8 \pm 3.5^{*, \dagger}$ \\
CLS & $23.7 \pm 2.8$ & $33.6 \pm 4.7^{*}$ & $72.2 \pm 3.4^{*, \dagger}$ & $68.5 \pm 4.1^{*, \dagger}$
\end{tabular}

Data are presented as mean $\pm \mathrm{SD}$. Vehicle $=$ phosphate buffered saline (PBS) and dimethyl sulfoxide (DMSO) only; $\mathrm{FeCl}_{3}=10 \mu \mathrm{mol} / / \mathrm{FeCl}_{3}$; carbon monoxidereleasing molecule-2 (CORM-2) $=100 \mu \mathrm{mol} / \mathrm{l}$ CORM-2. CGT, clot growth time (min); CLS, clot lifespan ( $\mathrm{min}$ ), the sum of CGT and CLT; CLT, clot lysis time (min); MRL, maximum rate of lysis (-dynes $/ \mathrm{cm}^{2}$ per s); MRTG, maximum rate of thrombus generation, the maximum velocity of clot growth observed (dynes $/ \mathrm{cm}^{2}$ per s); TMRL, time to maximum rate of lysis (min); TMRTG, time to maximum rate of thrombus generation, the time interval $(\mathrm{min})$ observed prior to maximum speed of clot growth; TTG, total thrombus generation, the total area under the velocity curve during clot growth (dynes $/ \mathrm{cm}^{2}$ ). ${ }^{*} P<0.05$ vs. vehicle. ${ }^{\dagger} P<0.05$ vs. $\mathrm{FeCl}_{3}$. ${ }^{\ddagger} P<0.05$ vs. CORM-2. 
Fig. 2
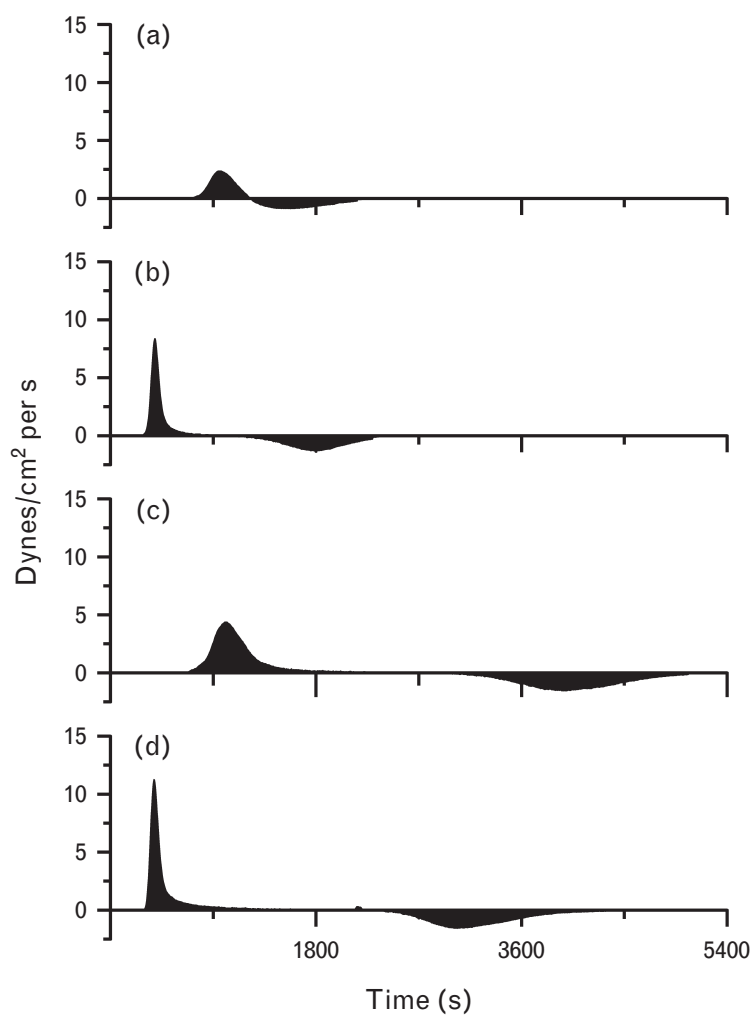

Effects of $\mathrm{FeCl}_{3}$ and carbon monoxide-releasing molecule-2 (CORM-2) on fibrinolytic kinetics in normal plasma. Plasma was exposed to (a) phosphate buffered saline (PBS) and dimethyl sulfoxide (DMSO), (b) DMSO and $10 \mu \mathrm{mol} / / \mathrm{FeCl}_{3}$, (c) PBS and $100 \mu \mathrm{mol} / / \mathrm{CORM}-2$, or (d) $10 \mu \mathrm{mol} / / \mathrm{FeCl}_{3}$ and $100 \mu \mathrm{mol} / / \mathrm{CORM}-2$ before addition of $100 \mathrm{U} / \mathrm{ml}$ of tissue-type plasminogen activator.

combination of $\mathrm{FeCl}_{3}$ and CORM-2 resulted in TTG values not different from CORM-2-exposed plasma. As for the onset of maximum clot lysis, exposure to $\mathrm{FeCl}_{3}$ or CORM-2 resulted in significantly prolonged TMRL values compared with unexposed plasma. Interestingly, exposure to $\mathrm{FeCl}_{3}$ and CORM-2 resulted in TMRL values significantly greater than those with $\mathrm{FeCl}_{3}-$ exposed plasma, but significantly less than CORM-2exposed plasma. With regard to velocity of clot lysis, addition of $\mathrm{FeCl}_{3}$ or CORM-2 resulted in significantly greater MRL, CORM-2 addition significantly increasing MRL compared with any condition in which it was not added. As for CGT, the addition of $\mathrm{FeCl}_{3}$ did not significantly affect CGT compared with unexposed plasma, whereas addition of CORM-2 significantly prolonged CGT compared with unexposed or $\mathrm{FeCl}_{3}-$ exposed samples. With regard to $\mathrm{CLT}, \mathrm{FeCl}_{3}$ or CORM-2 exposure significantly increased CLT compared with unexposed sample values, but the CLT values of samples exposed to $\mathrm{FeCl}_{3}$ and CORM-2 were not different from samples exposed to CORM-2 alone. Finally, concerning CLS, $\mathrm{FeCl}_{3}$ or CORM-2 exposure significantly increased CLS compared with unexposed sample values, but the CLS values of samples exposed to $\mathrm{FeCl}_{3}$ and CORM-2 were not different from samples exposed to CORM-2 alone.

\section{Scanning electron micrographic data}

The results of the experiments involving SEM are displayed in Fig. 3 (low magnification, $40000 \times$ ) and Fig. 4 (high magnification, $100000 \times$ ). Figure 3 a shows a typical fibrin network appearing like a net, with mostly major, thick fibrin fibers (shown with arrow). When $\mathrm{FeCl}_{3}$ is added to PRP followed by the addition of thrombin, a denser net is formed, with a finer fiber net developing (shown with * on micrograph) and thickened denser areas (shown with arrow), where the net fuses into a matted deposit (Fig. 3b). Exposure of PRP to CORM-2, with the addition of thrombin, resulted in a fused layer of fibrin fibers, wherein individual thick fibers could still be identified (Fig. 3c). In the final experiment, in which PRP was exposed to both $\mathrm{FeCl}_{3}$ and CORM-2, with the addition of thrombin, a homogenous matted layer is seen (shown with $*$ ), but now with thick fibers fused to form a near homogenous layer (shown with arrow) (Fig. 3d).

When magnification is increased, the ultrastructure seen above, is confirmed. Additionally, at a high machine magnification of $100000 \times$, we noted that the fibrin fibers, exposed to CORM-2, have globular plasma protein deposits that seem to form part of the fiber structure (arrows in Fig. 4c and d). These deposits are not present in healthy fibrin or fibrin exposed to iron (Fig. $4 \mathrm{a}$ and b). This might suggest that CORM-2 affects the fibrin packaging.

\section{Discussion}

The primary finding of the present investigation was that iron and carbon monoxide appear to enhance coagulation and diminish fibrinolysis by two separate and mostly complementary mechanisms. The viscoelastic and SEM data demonstrated three different kinetic and structural effects of $\mathrm{FeCl}_{3}, \mathrm{CORM}-2$, and $\mathrm{FeCl}_{3}$ combined with CORM-2 on thrombus formation. $\mathrm{FeCl}_{3}$ hastened the onset and increased the velocity of clot formation in both normal and FXIII-deficient plasma, with a relatively greater increase in FXIII-deficient plasma. Thus, iron may compete to a certain extent with FXIII-dependent cross-linking. In contrast, CORM-2 somewhat delayed the onset of biophysical engagement of fibrin polymers, yet increased the velocity of clot formation and even more so strength, in both normal and FXIII-deficient plasma. Critically, combining $\mathrm{FeCl}_{3}$ and CORM-2 resulted in thrombus formation that incorporated all the hypercoagulable features of either molecule. The ultrastructural correlate of these conditions demonstrated a unique pattern following $\mathrm{FeCl}_{3}$ exposure that was very different from that observed after CORM-2 exposure, with thrombi exposed to both molecules displaying features of both separate exposures. Particularly, 
Fig. 3
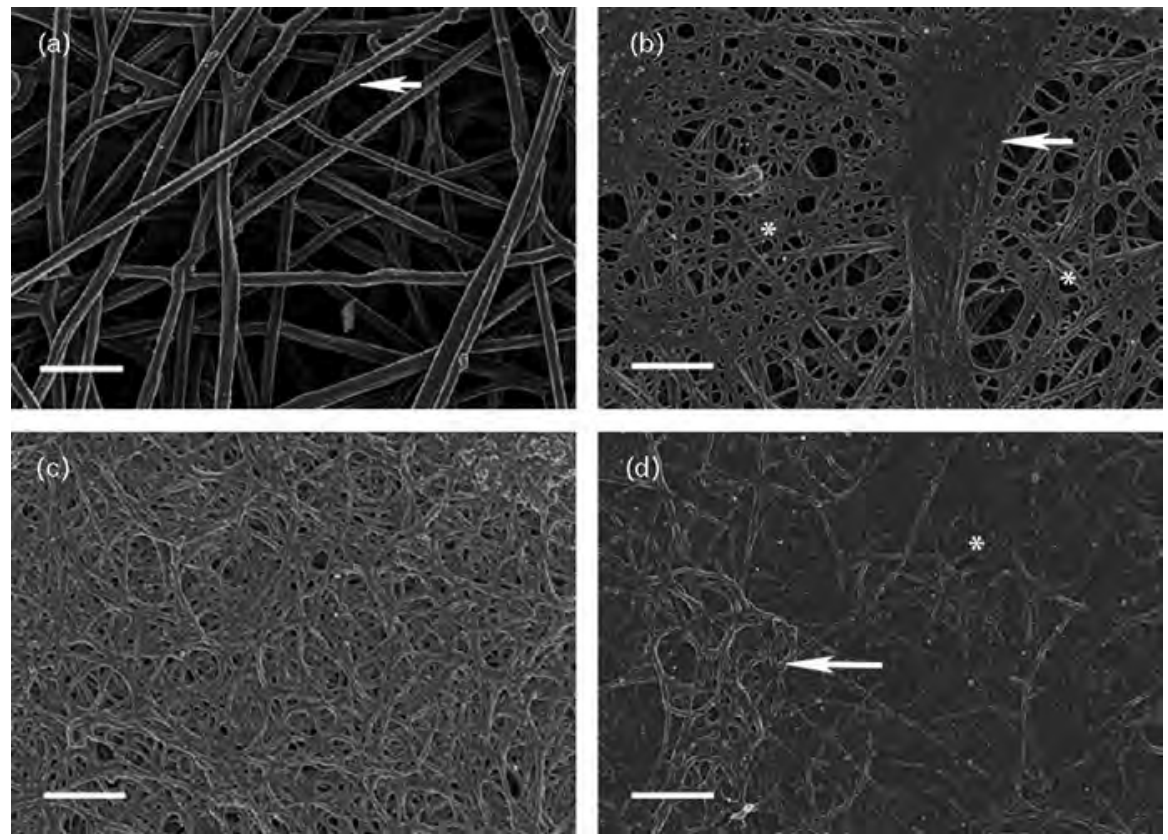

Scanning electron microphotographs of platelet-rich plasma (PRP) after exposure to $\mathrm{FeCl}_{3}$ or/and carbon monoxide-releasing molecule-2 (CORM-2) at low magnification. (a) PRP and thrombin; arrow shows thick, major fibrin fiber. (b) PRP and $\mathrm{FeCl}_{3}$ and thrombin arrow shows matted and fused fibrin fiber deposit; * shows a finer fibrin fiber net compared to the healthy PRP net, seen in (a); (c) PRP and CORM-2 and thrombin; the resulting fibrin net shows a homogenous, denser fiber layer. (d) PRP with $\mathrm{FeCl}_{3}$ and $\mathrm{CORM}-2$ and thrombin, a near homogenous layer (*) with fused fibers (arrow) is seen. All micrographs taken at $40000 \times$ machine magnification. Scale $=1 \mu \mathrm{m}$.

the combination of CORM-2 and iron (as shown in Figs. 3d and 4d) shows the most complex ultrastructure, in which the combination of the compounds with PRP causes a near homogenous layer. In sum, these data support the concept that iron and carbon monoxide enhance the substrate properties of fibrinogen by separate mechanisms.

With regard to the effects of $\mathrm{FeCl}_{3}$ and CORM-2 on fibrinolysis, it appeared that CORM-2-mediated effects overshadowed those of $\mathrm{FeCl}_{3}$. Although exposure to $\mathrm{FeCl}_{3}$ prolonged CLT and CLS compared with unexposed plasma, the hypofibrinolytic effects of the combination of $\mathrm{FeCl}_{3}$ and CORM-2 were no different than CORM-2 alone. Although the exact mechanism by which $\mathrm{FeCl}_{3}$ diminished fibrinolysis was not discerned by the present investigation, the viscoelastic-derived kinetic data would suggest that the biochemical mechanisms [13] responsible for carbon monoxide-mediated antifibrinolytic actions are more important than $\mathrm{FeCl}_{3}$-mediated changes in fibrinogen polymerization. Future investigations of $\mathrm{FeCl}_{3}$-mediated hypofibrinolysis will discern the specific mechanisms, but are outside the scope of the present investigation.

There are potential clinical implications of our findings. Given that both iron and carbon monoxide are products of HO-1 activity, it is likely that they coexist in various micromolar concentrations in conditions such as cancer [27-31], diabetes mellitus [21], rheumatoid arthritis [22], and mechanical circulatory support $[18,26]$. Although it is not possible to directly extrapolate our findings as the underpinning of hypercoagulability in these various settings $[18,22,26-31]$, it is possible that exposure of circulating fibrinogen to various combinations of iron and carbon monoxide may be enhancing coagulation in these disease states.

It is important to carefully note that the two approaches used in this investigation are quite different, and direct comparisons or conclusions made about the biochemical and biophysical interactions of iron and carbon monoxide in thrombus formation should be system specific. On one hand, Dr Nielsen's viscoelastic studies employed weak contact activation (thrombelastographic cup and pin) in recalcified, previously frozen, platelet poor plasma obtained from either a normal pool of 30 or more donors or a single FXIII-deficient donor. This system was optimized to detect coagulation and fibrinolytic kinetic changes. On the other hand, PRP from three female donors was obtained and immediately used by E.P., with the plasma kept in an anticoagulated state with sodium citrate addition and coagulation caused by thrombin addition, which causes clot formation in a calcium-independent fashion. It was never our intent 
Fig. 4
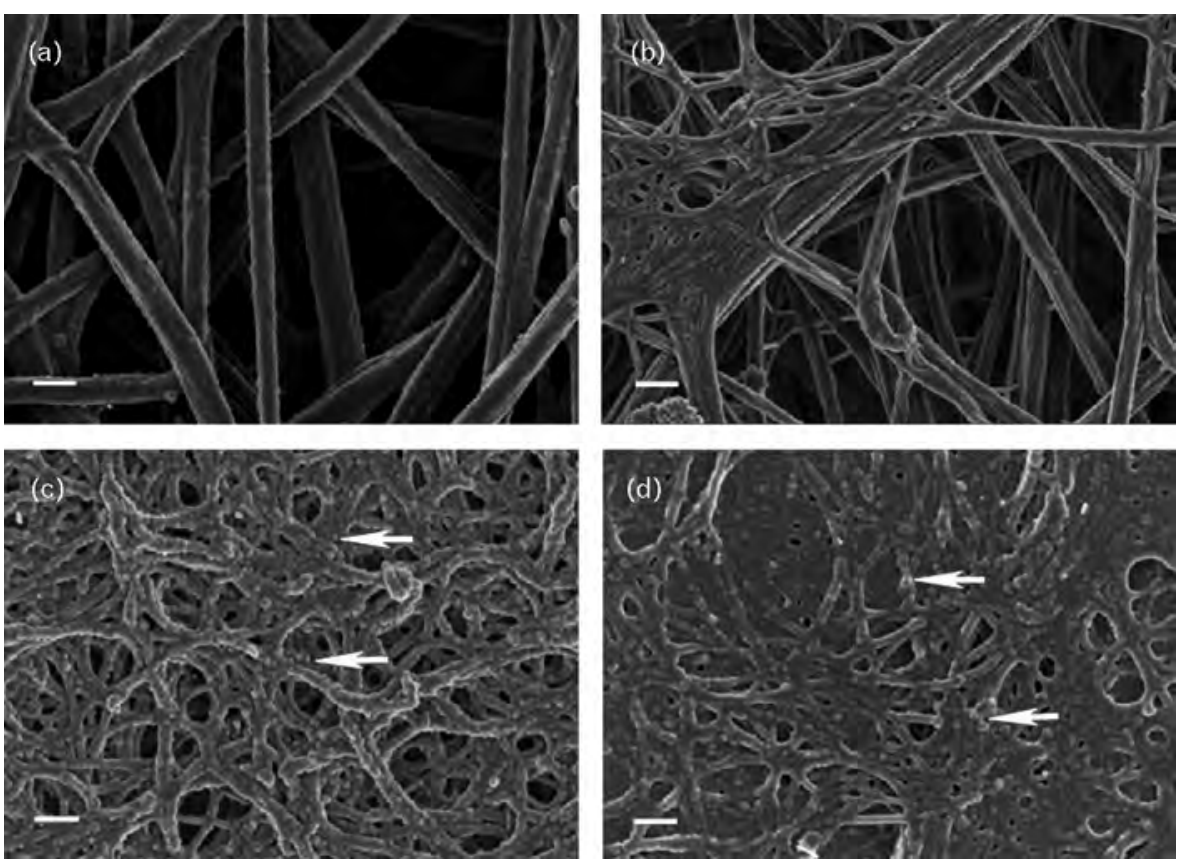

Scanning electron microphotographs of platelet-rich plasma (PRP) after exposure to $\mathrm{FeCl}_{3}$ or/and carbon monoxide-releasing molecule-2 (CORM-2) at high magnification. (a) PRP and thrombin. (b) PRP and $\mathrm{FeCl}_{3}$ and thrombin. (c) PRP and CORM-2 and thrombin; arrows show globular plasma protein deposits. (d) PRP with $\mathrm{FeCl}_{3}$ and CORM-2 and thrombin; arrows show globular plasma protein deposits. All micrographs taken at $100000 \times$ machine magnification. Scale $=200 \mathrm{~nm}$.

to completely parallel all aspects of experimentation; instead, only the four experimental conditions (e.g. control, carbon monoxide addition, iron addition, carbon monoxide + iron addition) were to be consistent. The concentrations of carbon monoxide and iron used and the rationale for the concentrations of each have already been mentioned in the previously presented Methods section. Although some may say a lack of complete duplication of methodology within the two experimental systems is a weakness that should make one take pause when making conclusions, we would have to differ with them. Instead, despite the obvious differences in materials, plasmas, and experimental endpoints (e.g. kinetics vs. ultrastructure), we nevertheless demonstrated four different kinetic and ultrastructural profiles that indicated that iron and carbon monoxide affect coagulation by different mechanisms that positively interact to form a faster, stronger, more intricate thrombus. In sum, it is this teleological similarity between the two systems that lends strength to our conclusions rather than weakness of support secondary to lack of complete material parallelism.

The present investigation has limitations. First, concentrations of both $\mathrm{FeCl}_{3}$ and CORM-2 were utilized to provide robust viscoelastic and SEM results rather than generating a broad range of the effects of various concentrations of these molecules separately or in combination. It was also never our intent to simulate 'physiological' concentrations of iron or carbon monoxide in our investigation. However, we used this approach to maximize the identification of interactions of iron and carbon monoxide in both viscoelastic and SEM systems, and our results can now serve as the rational basis for more incremental and expansive investigation of concentration-effect relationships. Second, experimentation was plasma-based, and in vitro in its approach. Again, such an approach was aimed at identifying iron and carbon monoxide interactions and discerning whether these two molecules modified coagulation and fibrinolysis by different mechanisms. It is anticipated that in-vivo validation, perhaps with the aforementioned rabbit model $[15,16]$, will be performed to determine whether combinations of iron and carbon monoxide diminish bleeding time. Taken as a whole, although this investigation has limitations, it will serve as a foundation for more extensive investigation of the interaction of iron and carbon monoxide in coagulation and fibrinolysis.

In conclusion, we present the first evidence that iron and carbon monoxide interact with key coagulation and fibrinolytic processes. Both viscoelastic and SEM data demonstrate distinct kinetic and ultrastructural profile effects, respectively, of iron, carbon monoxide, and their combination on thrombus formation. Future in-vitro and in-vivo investigations of these phenomena are planned, 
especially in settings that may involve upregulation of HO-1 activity $[18,22,26-31]$.

\section{Acknowledgements}

This investigation was supported by the Departments of Anesthesiology and Physiology.

\section{Conflicts of interest}

The authors declare no conflicts of interest.

\section{References}

1 Lipinski B. Modification of fibrin structure as a possible cause of thrombolytic resistance. J Thromb Thrombolysis 2010; 29:296-298.

2 Lipinski B, Pretorius E. Novel pathway of iron-induced blood coagulation: implications for diabetes mellitus and its complications. Pol Arch Med Wewn 2012; 122:115-122.

3 Lipinski B, Pretorius E, Oberholzer HM, van der Spuy WJ. Iron enhances generation of fibrin fibers in human blood: implications for pathogenesis of stroke. Microsc Res Tech 2012; 75:1185-1190.

4 Pretorius E, Vermeulen N, Bester J, Lipinski B. Novel use of scanning electron microscopy for detection of iron-induced morphological changes in human blood. Microsc Res Tech 2013; 76:268-271.

5 Lipinski B, Pretorius E. Iron-induced fibrin in cardiovascular disease. Curr Neurovasc Res 2013; 10:269-274.

6 Pretorius E, Vermeulen N, Bester J, Lipinski B, Kell DB. A novel method for assessing the role of iron and its functional chelation in fibrin fibril formation: the use of scanning electron microscopy. Toxicol Mech Methods 2013; 23:352-359.

7 Pretorius E, Lipinski B. Differences in morphology of fibrin clots induced with thrombin and ferric ions and its pathophysiological consequences. Heart Lung Circ 2013; 22:447-449.

8 Nielsen VG, Kirklin JK, George JF. Carbon monoxide releasing molecule-2 increases the velocity of thrombus growth and strength in human plasma. Blood Coagul Fibrinolysis 2009; 20:377-380.

9 Nielsen VG, Kirklin JK, George JF, Messinger JD. Carbon monoxide releasing molecule-2 decreases thick diameter fibrin fibre formation in normal and factor XIII deficient plasma. Blood Coagul Fibrinolysis 2010 21:101-105.

10 Machovec KA, Ushakumari DS, Welsby IJ, Nielsen VG. The procoagulant properties of purified fibrinogen concentrate are enhanced by carbon monoxide releasing molecule-2. Thromb Res 2012 129:793-796.

11 Nielsen VG, Cohen JB, Malayaman SN, Nowak M, Vosseller K. Fibrinogen is a heme-associated, carbon monoxide sensing molecule: a preliminary report. Blood Coagul Fibrinolysis 2011; 22:443447.

12 Nielsen VG, Kirklin JK, George JF. Carbon monoxide releasing molecule-2 (CORM-2) decreases fibrinolysis in human plasma. Blood Coagul Fibrinolysis 2009; 20:448-455.

13 Arkebauer MR, Kanaparthy SS, Malayaman SN, Vosseller K, Nielsen VG. Carbon monoxide and nitric oxide modulate alpha-2-antiplasmin and plasmin activity: role of heme. Blood Coagul Fibrinolysis 2011; 22:712719.
14 Nielsen VG, Arkebauer MR, Vosseller K. Redox-based thrombelastographic method to detect carboxyhemefibrinogen mediated hypercoagulability. Blood Coagul Fibrinolysis 2011; 22:657-661.

15 Nielsen VG, Chawla N, Mangla D, Gomes SB, Arkebauer MR, Wasko KA, et al. Carbon monoxide releasing molecule-2 enhances coagulation in rabbit plasma and decreases bleeding time in clopidogrel/aspirin treated rabbits. Blood Coagul Fibrinolysis 2011; 22:756-759.

16 Nielsen VG, Arkebauer MR, Wasko KA, Malayaman SN, Vosseller K. Carbon monoxide releasing molecule-2 decreases fibrinolysis in vitro and in vivo in the rabbit. Blood Coagul Fibrinolysis 2012; 23:104-107.

17 Balla J, Vercellotti GM, Jeney V, Yachie A, Varga Z, Eaton JW, Balla G. Heme, heme oxygenase and ferritin in vascular endothelial cell injury. Mol Nutr Food Res 2005; 49:1030-1043.

18 Nielsen VG, Pearson T, Smith MC. Increased carbon monoxide production by hemeoxygenase- 1 caused by device-mediated hemolysis: thrombotic phantom menace? Artif Organs 2013; 37:1008-1014.

19 Lipinski B, Pretorius E. Hydroxyl radical-modified fibrinogen as a marker of thrombosis: the role of iron. Hematology 2012; 17:241-247.

20 Orino K. Functional binding analysis of human fibrinogen as an iron- and heme-binding protein. Biometals 2013; 26:789-794.

21 Bao W, Song F, Li X, Rong S, Yang W, Zhang M, et al. Plasma heme oxygenase- 1 concentration is elevated in individuals with type 2 diabetes mellitus. PLoS One 2010; 5:e12371.

22 Takhashi A, Mori M, Naruto T, Nakajima S, Miyamae T, Imagawa T, Yokota $\mathrm{S}$. The role of heme oxygenase-1 in systemic-onset juvenile idiopathic arthritis. Mod Rheumatol 2009; 19:302-308.

23 Pretorius E, Oberholzer HM, van der Spuy WJ, Swanepoel AC, Soma P. Qualitative scanning electron microscopy analysis of fibrin networks and platelet abnormalities in diabetes. Blood Coagul Fibrinolysis 2011; 22:463-467.

24 Pretorius E, Oberholzer HM, van der Spuy WJ, Swanepoel AC, Soma P. Scanning electron microscopy of fibrin networks in rheumatoid arthritis: a qualitative analysis. Rheumatol Int 2012; 32:1611-1615.

25 Nielsen VG, Hafner DT, Steinbrenner EB. Tobacco smoke induced hypercoagulation in human plasma: role of carbon monoxide. Blood Coagul Fibrinolysis 2013; 24:405-410.

26 Smith MC, Nielsen VG. Detection of carboxyhemefibrinogen and methemefibrinogen in a patient with thrombosis of a Heartmate ll ventricular assist device. ASAlO J 2013; 59:93-95.

27 Nielsen VG, Garol BD, Zelman EA, Guerrero MA. Hemeoxygenase-1 mediated hypercoagulability in a patient with thyroid cancer. Blood Coagul Fibrinolysis 2013; 24:663-665.

28 Nielsen VG, Ley MLB, Waer AL, Alger PW, Matika RW, Steinbrenner EB. Plasmatic hypercoagulation in patients with breast cancer: role of hemeoxygenase-1. Blood Coagul Fibrinolysis 2013; 24:809-813.

29 Nielsen VG, Lemole GM Jr, Weinand ME, Baaj AA, Hussaini S, Steinbrenner EB. Brain tumors enhance plasmatic coagulation: role of hemeoxygenase-1. Anesth Analg (in press).

30 Nielsen VG, Gharagozloo F, Matika RW, Kim S, Zelman EA, Steinbrenner EB. Lung tumor effects on plasmatic coagulation: role of hemeoxygenase1. Lung Cancer 2014; 83:288-291.

31 Nielsen VG, Nfonsam VN, Matika RW, Ong ES, Jie T, Warneke JA, Steinbrenner EB. Colon and pancreas tumors enhance plasma coagulation kinetics: role of hemeoxygenase-1. Blood Coagul Fibrinolysis (in press).

32 Pretorius E, Oberholzer HM, van der Spuy WJ, Meiring JH. The changed ultrastructure of fibrin networks during use of oral contraception and hormone replacement. J Thromb Thrombolysis 2010; 30:502-506. 